\title{
The in-flight background of IBIS/PICsIT ${ }^{\star}$
}

\author{
J. B. Stephen ${ }^{1}$, E. Caroli ${ }^{1}$, A. Malizia ${ }^{1}$, L. Natalucci ${ }^{2}$, L. Bassani ${ }^{1}$, and G. Di Cocco ${ }^{1}$ \\ ${ }^{1}$ Istituto di Astrofisica Spaziale e Fisica Cosmica Sezione di Bologna, Area di Ricerca di Bologna, via P. Gobetti 101, Bologna, \\ Italy \\ 2 Istituto di Astrofisica Spaziale e Fisica Cosmica Area di Ricerca di Roma 2/Tor Vergata, via del Fosso del Cavaliere, 100, \\ Roma, Italy
}

Received 15 July 2003 / Accepted 18 September 2003

\begin{abstract}
The PICsIT instrument is the high-energy imaging detector of IBIS on board INTEGRAL and comprises 4096 individual scintillator elements each working in the energy range from about $175 \mathrm{keV}$ to $10 \mathrm{MeV}$. Images of the sky are created using the coded aperture technique wherein each point in the detection plane contributes to the final image. For this reason the sensitivity at any point in the image is highly dependent on both the total background and its spatial and temporal uniformity. Herein we describe the PICsIT in-flight background, for the principal modes of operation, from the point of view of total count rate and spectrum, and discuss both the spatial and temporal stability.
\end{abstract}

Key words. instrumentation: detectors; telescopes - methods: data analysis

\section{Introduction}

The PICsIT instrument is the high-energy plane of the IBIS imaging telescope on INTEGRAL. It consists of 4096 individual CsI scintillation crystals, each coupled to a photodiode for the read-out of the light produced by an interacting gamma-ray. A complete description of the instrument can be found elsewhere in this volume (Labanti et al. 2003). Briefly, the detection plane comprises 16 semi-modules, each of $16 \times 16$ pixels. Each group of $16(4 \times 4)$ pixels is read out electronically by one ASIC. The semi-modules are arranged in pairs as 8 modules which are then placed in a $2 \times 4$ format to create a $64 \times 64$ pixel detection plane. There is a gap of one pixel between neighbouring modules leading to an effective dimension of the detection plane of $65 \times 67$ pixels. Around the detection plane are placed 16 blocks of BGO scintillator which act as a veto system.

The IBIS imager uses the technique of coded aperture imaging in order to produce images of the sky (see Goldwurm et al. 2003 for an in-depth discussion of the IBIS data analysis techniques). This entails integrating over time to create a count map (or shadowgram) of recorded events. The shadowgram consists of many overlapping shadows of the coded aperture, one for each source in the field of view, superimposed on a background. This shadowgram is then the input into an

Send offprint requests to: J. B. Stephen,

e-mail: stephen@bo.iasf.cnr.it

* Based on observations with INTEGRAL, an ESA project with instruments and science data centre funded by ESA member states (especially the PI countries: Denmark, France, Germany, Italy, Switzerland, Spain), Czech Republic and Poland, and with the participation of Russia and the USA. algorithm used for producing the final image. For the fully coded field of view, all the data collected are involved in a weighted addition process for every point in the reconstructed image, and in order for this technique to be satisfactory, the background in the recorded shadowgram must be as low and as spatially uniform as possible (or at least, all the sources of disuniformity must be known and calibrated beforehand and accounted for either through the weighting procedure or through some other correction process). For the standard mode of operation, the accumulation of the shadowgrams is performed onboard over a standard timescale of $2150 \mathrm{~s}$ (programmable), one for single events and another for multiple pixel interactions. The data is transmitted to ground as sets of $64 \times 64 \times 256$ elements corresponding to a 256 -channel spectrum for each pixel. In this mode of operation it is therefore not possible to return to an event list to make subsequent gain/offset corrections onground. In some cases it is possible to send the data to ground as complete photon lists (again, one for single site interactions and another for multiple events), however due to telemetry limitations only about one third of the events can be transmitted.

It is clear that the design of the detection plane allows itself to the creation of systematic spatial disuniformities in background due to the differing local conditions of the individual pixels. In particular there are natural frequencies at 4 (ASIC), 16 (semimodule) and 32 (module) pixels. A further discussion of the systematic effects induced by spatial non-uniformity in both detection planes of the IBIS telescope is available elsewhere in this volume (Natalucci et al. 2003). In the following sections we will describe the background in the PICsIT standard imaging mode in terms of the total count rate, the spectral 

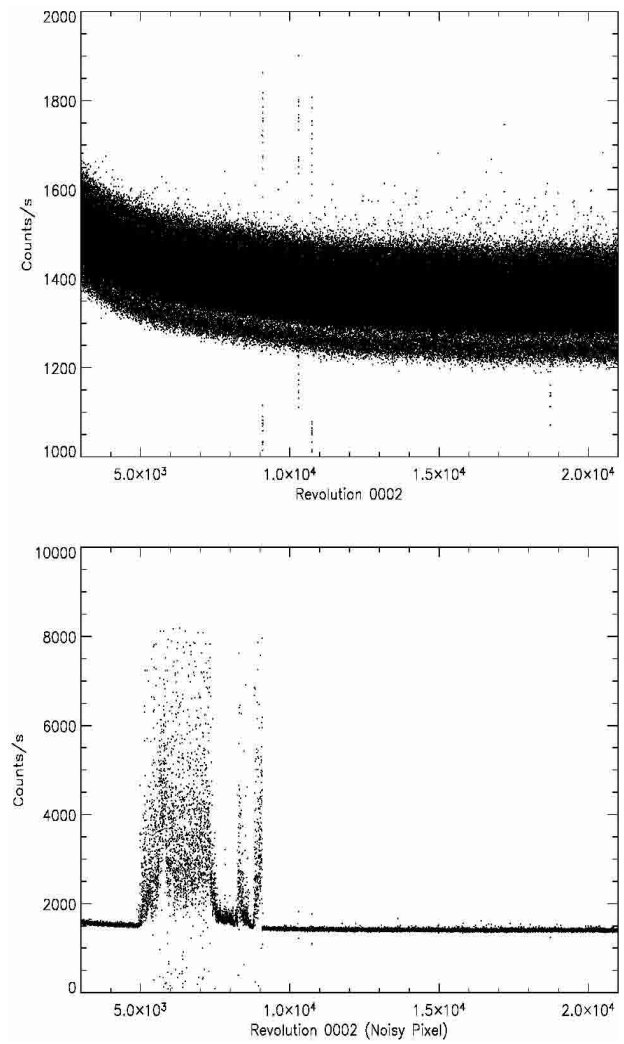

Fig. 1. The evolution in detection plane trigger rate with time from switch on during revolution 2. The top panel is a superimposition of 15 semi-modules, while the bottom panel shows the remaining semimodule in which a pixel becomes noisy. To date, only 4 pixels have been switched off due to becoming noisy during flight adding to the 50 already killed before launch.

form, spatial non-uniformities and the background stability in time.

\section{The PICsIT ratemeters}

Each PICsIT semi-module has a ratemeter whose value is transmitted to ground in the housekeeping format every $8 \mathrm{~s}$. Figure 1 shows the count rate for the individual PICsIT semi-modules from switch on at the beginning of revolution 2. The top panel shows the superimposition of 15 semi-modules while the bottom panel shows one semimodule in which a pixel becomes noisy. Pixels which become noisy in PICsIT are treated in a different fashion from the ISGRI plane in that they are killed definitively. Before launch 50 pixels had been identified as being potentially noisy and were switched off. At the time of writing ( +8 months) only 4 more have had to be killed.

The total count rate per semi-module can be seen to decrease in an exponential manner from around $1500 \mathrm{c} / \mathrm{s}$ (or $24000 \mathrm{c} / \mathrm{s}$ for the entire plane) with an extrapolated constant value of around $1300 \mathrm{c} / \mathrm{s}(\sim 21000 \mathrm{c} / \mathrm{s})$. During the following weeks, until around the middle of November the orbit of INTEGRAL was adjusted to attain the final orbit, raising the perigee and thereby decreasing the radiation activation during the perigee passages. At the beginning of November the veto modules were switched on and their parameters optimised.

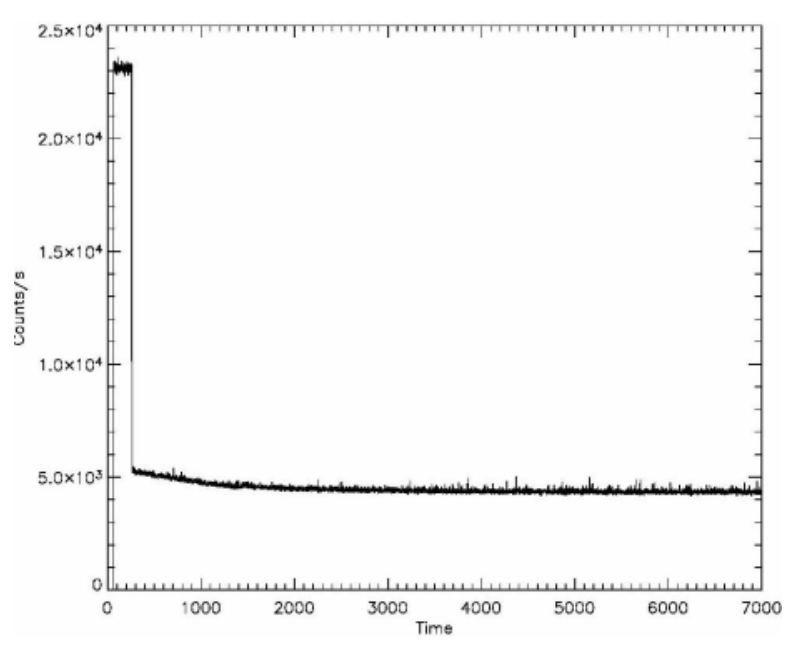

Fig. 2. The integrated ratemeters for the entire PICsIT detection plane for a blank field observation during revolution 38 . The time axis is in housekeeping packet cycle time ( $8 \mathrm{~s})$, thus $56 \mathrm{ks}$ of data are shown. The high count rate at the beginning corresponds to where the veto was off.

Figure 2 shows the integrated PICsIT ratemeters for revolution 38 (February 2003) during a blank sky field observation. It can be seen that the vetoed count rate is relatively uniform throughout the orbit at a rate of $4250 \mathrm{c} / \mathrm{s}$ while for the few periods where the veto was switched off the count rate was about $23000 \mathrm{c} / \mathrm{s}$ in line with the constant value extrapolated above. This trigger rate, after thresholding and event selection, eventually gives rise to countrates of around 2700 counts/s single events and 600 counts/s multiple events.

\section{PICsIT science data}

The standard mode of operation of PICsIT (Spectral Imaging) consists of the integration aboard of data histograms. Each data cube consists of a $64 \times 64 \times 256$ set of counts corresponding to the 4096 pixels and 256 energy channels. There are two of these blocks per integration - one for single site events and the other for multiple energy deposits. While the energy of the individual events is digitised at 10-bit precision, this resolution is degraded to 8-bit for transmission. The energy range for single events covers the range up to about $7 \mathrm{MeV}$ and for multiple events up to around $14 \mathrm{MeV}$. In addition to these data blocks a data stream of counts is produced with very limited energy resolution and no spatial resolution (Timing mode). This data will not be discussed here.

\subsection{Spectral distribution}

The integrated spectra of both single and multiple events are shown in Fig. 3 for the blank field observation of revolution 38. In addition to the standard mode data the photon-by-photon mode (PPM) singles data for an observation immediately beforehand is superimposed.

Some broad features at $511 \mathrm{keV}$ and $662 \mathrm{keV}$ are evident, as well as an excess around 1-2 MeV and some evidence for lines at around 2 and $3 \mathrm{MeV}$. All these features were predicted by 


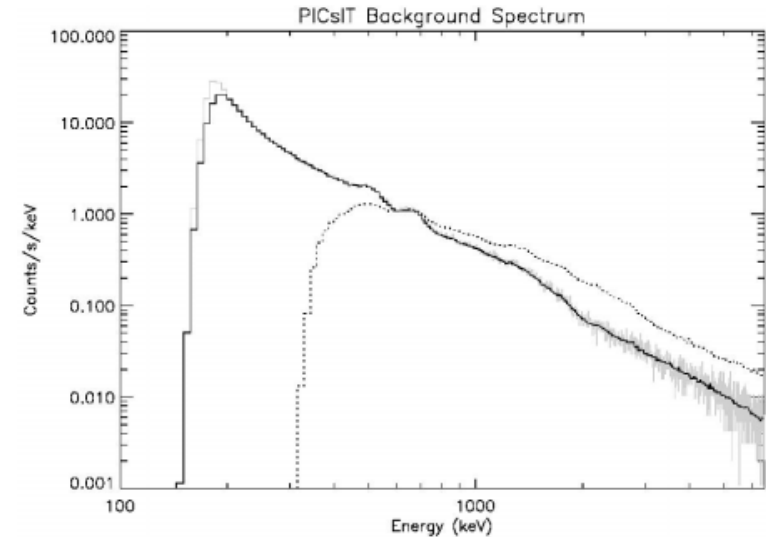

Fig. 3. The spectrum for the entire PICsIT detection plane for blank sky field observations in revolution 38 . The single event spectra for both PPM (light) and Histogram (dark) modes are shown, while the multiple event spectrum (dotted) is for the histogram mode only. The main features present are at 511 and $662 \mathrm{keV}$ with smaller excesses between 1 and $3 \mathrm{MeV}$.

the INTEGRAL Mass Model (TIMM) calculations (Ferguson et al. 2003), although the overall intensity is around a factor of two higher. The multiple events outnumber the single site interactions above the $662 \mathrm{keV}$ line. There is also an excess in background at lower energies $(<250-300 \mathrm{keV})$. The reason for this is phosphorescence of the CsI scintillators after activation by charged particle interactions. This phenomenon is described in detail by Segreto et al. (2003). Although it is possible to eliminate this component in the photon-by-photon event list data, in the pre-accumulated histograms there is nothing that can be done to reduce this component and so the background shown in the figure is that which determines the statistical limit to the sensitivity of PICsIT in its standard operating mode.

\subsection{Spatial distribution}

As described above, the modular design of the PICsIT instrument is responsible for a large amount of spatial nonuniformity in the distribution of the background count rate. Figures 4 and 5 show false-colour images of the detected events, both single and multiple, for two different energy ranges, together with the projection of these shadowgrams onto the $Y$ and $Z$ axes. Broadly speaking, it can be seen that the spatial distribution is more structured for the single events at higher energies $(>500 \mathrm{keV})$ than at lower. For multiple events there is a high degree of structure in both energy ranges but taking different forms. Common to both multiple and single events is the higher count rate in the top left hand and 3 bottom right hand modules.

The module borders are always visible for single events, while at the higher energies an ASIC-determined structure is clearly visible with adjacent pairs of columns and rows being alternately higher and lower. This is also evident to a lesser degree in the multiple event distribution. In Fig. 6 we show the single event spectra for the $2 \times 2$ ASIC centre pixels in comparison with that for the outer ring of 12 pixels as integrated over the entire detection plane. It is clear that there is an excess of
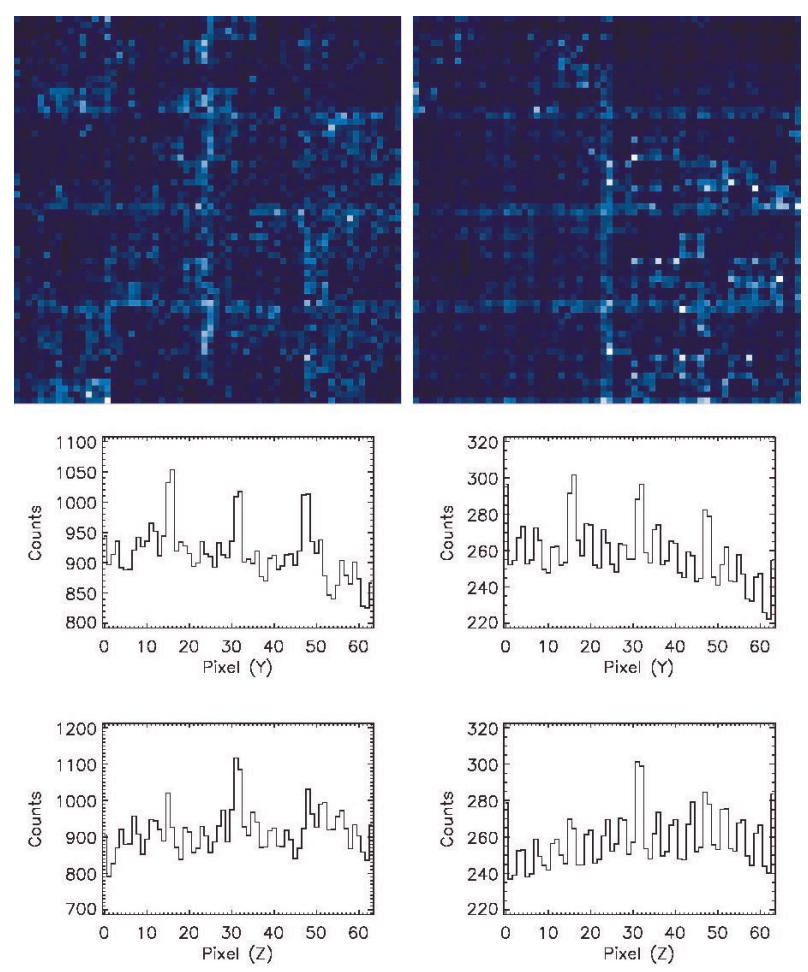

Fig. 4. The spatial distribution of the PICsIT single event background both below (left) and above $511 \mathrm{keV}$. Projections onto the $Y$ and $Z$-axis are also shown.

the latter over the former above around $\mathrm{MeV}$ which cannot be explained in terms of a pure gain and offset difference.

The well known sensitivity of the coded aperture technique to this type of spatial non-uniformity dictates that the spatial form of the background must be modelled to a high degree of accuracy (Malaguti et al. 2001) in order to reduce the systematic variation to a point where it will be possible to achieve a sensitivity close to the statistical limit.

\subsection{Time variation}

As described above, the standard mode of operation of PICsIT accumulates data over periods of around $2150 \mathrm{~s}$. It is clear that temporal variations in the background should not occur at timescales shorter than this basic period. Figure 7 shows the difference between two singles spectra obtained 21 days apart at the very beginning and end of the Crab calibration measurements expressed in terms of standard deviations from the mean spectrum. A very slight DC offset of about $0.5 \sigma$, corresponding to a difference in absolute counting rate is visible. It is clear however, that outside of the lowest energies where threshold and CsI phosphorescence events are important there is no appreciable difference between the two spectra. Of great importance in making this comparison is that although the spectra were obtained at a great difference in time, the temperature of the detection plane at both measurements was essentially the same. As is discussed in the analysis of the IBIS calibration source data (Malaguti et al. 2003), there is a gain change effect of approximately $0.3 \% /{ }^{\circ} \mathrm{C}$ for the scintillation elements in 

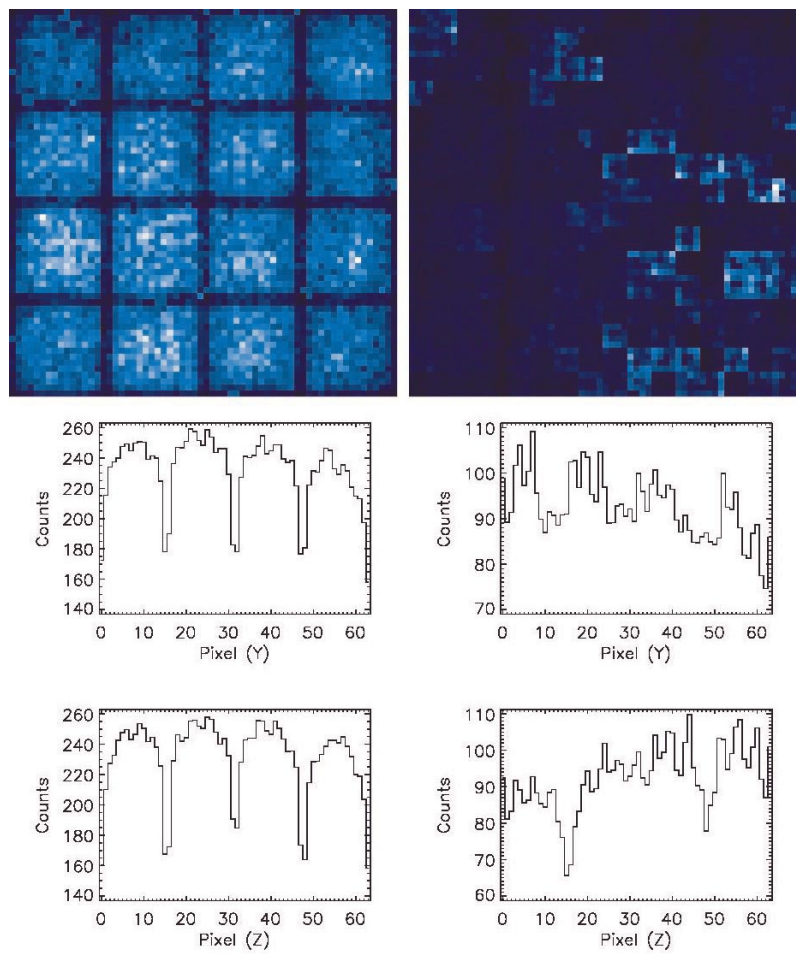

Fig. 5. As Fig. 4 but for multiple events, showing the distributions below (left) and above $1 \mathrm{MeV}$.

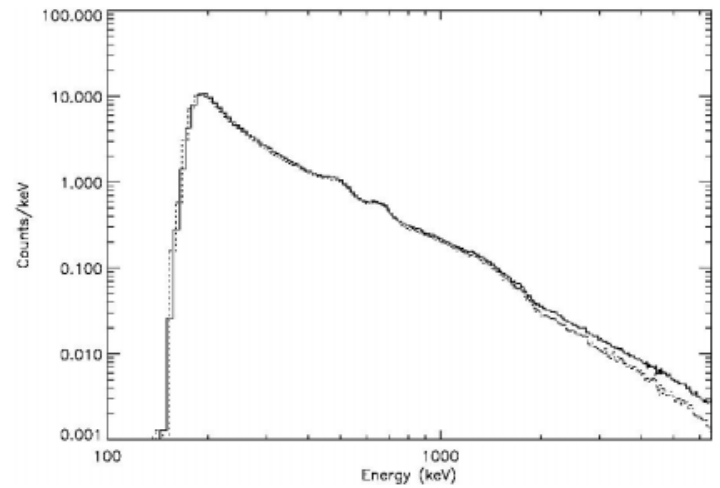

Fig. 6. The spectra corresponding to the data shown in Fig. 4. The lower spectrum is the sum of the ASIC centre pixels $(2 \times 2)$ while the upper curve is the sum of the ASIC outer pixels (12). It can be seen that there is a divergence in the spectra above approximately $2 \mathrm{MeV}$ which is not compatible with a difference in gain and offset.

the calibration source peaks, which will also be the case for the science data.

Figure 8 shows the temperature profile for revolutions 39 through 49. Revolutions 39-45 correspond to the Crab calibration observations (and to the period when the instrument was left on during perigee passage thus avoiding the cooling which would result if PICsIT was switched off). It is clear that there is a small cooling effect during the perigee passages, but the variations are of the order only $\pm 0.5{ }^{\circ} \mathrm{C}$ over a timescale of 21 days. In revolutions 46 to 49 the spacecraft is pointed at various sources and the differing aspect angle causes wide temperature fluctuations of up to 6 degrees in only 30 hours. The temperature change produces a large difference in the background

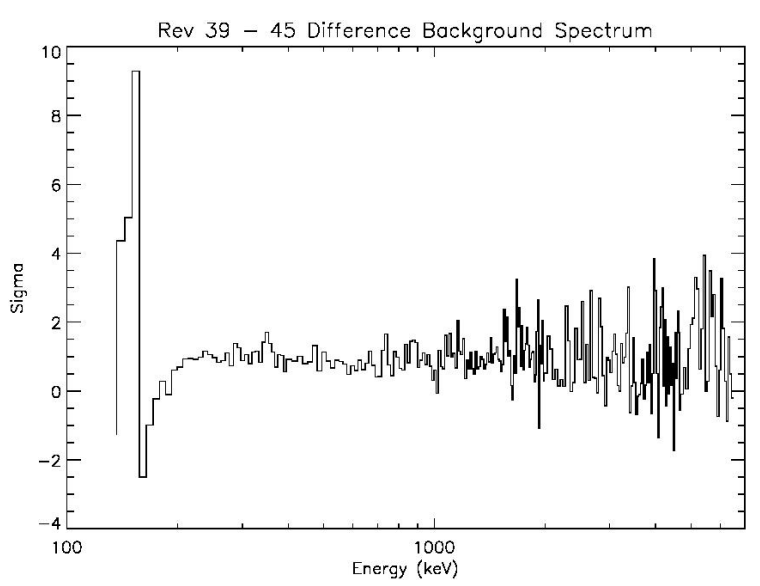

Fig. 7. The difference in the single event spectra from science windows in revolution 39 and revolution 45, corresponding to a time difference of about 21 days expressed in terms of standard deviation from the mean. Apart from a difference at low energies where noise effects are important, no appreciable change in the spectrum is apparent.
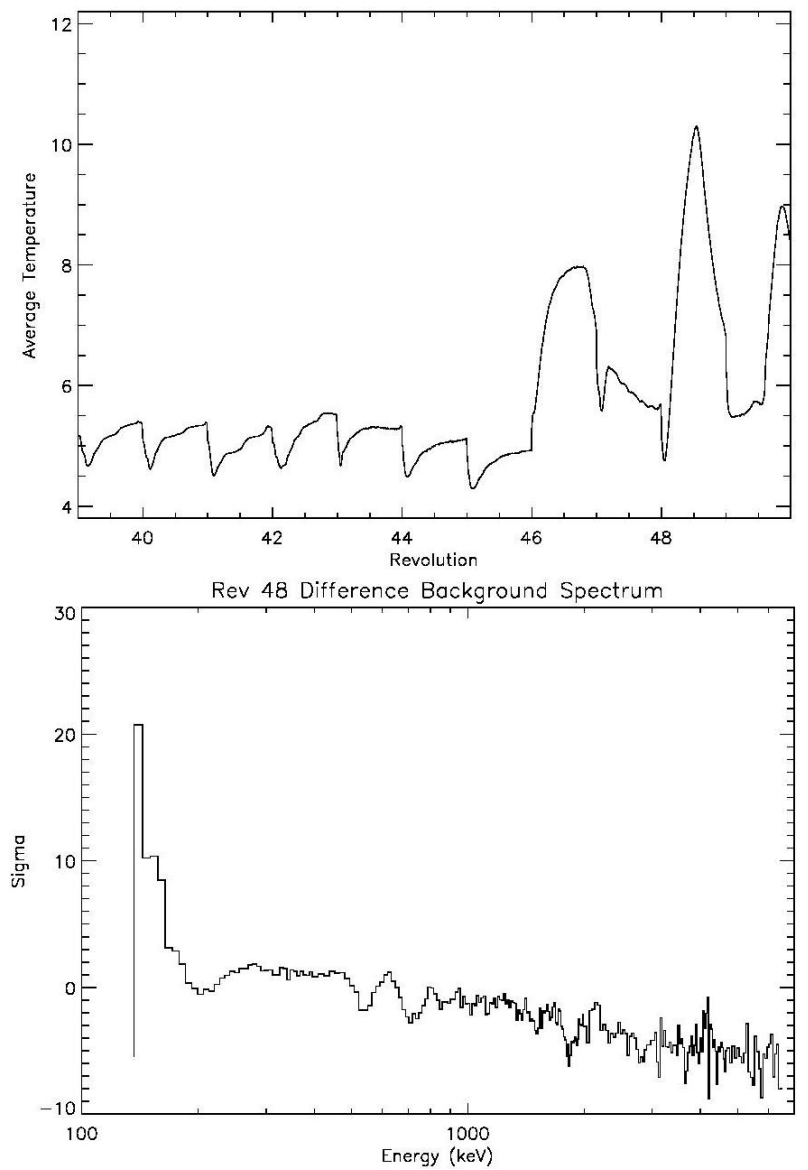

Fig. 8. (above) The average temperature of the PICsIT detection plane throughout revolutions 39 to 49. (below) The comparison of background spectra taken at the lowest and highest temperatures of revolution 48. A clear gain effect is obvious as demonstrated by the slope and features around the $511 \mathrm{keV}$ and $662 \mathrm{keV}$ lines.

spectrum as seen in the figure which must be compensated for. This difference is due to the change in light output from the CsI crystal with temperature leading to an effective gain change. 


\section{Conclusions}

The PICsIT instrumental background is highly structured spatially and must be modelled to a high degree of accuracy in order to obtain the maximum sensitivity. However, apart from the expected effects of temperature changes from solar aspect angle variations, it has shown itself to be stable in time.

Acknowledgements. The Italian participation in the INTEGRAL/IBIS project is financed by the Italian Space Agency (ASI).

\section{References}

Di Cocco, G., Caroli, E., Celesti, E., et al. 2003, A\&A, 411, L189 Ferguson, C., Barlow, E. J., Bird, A. J., et al. 2003, A\&A, 411, L19 Goldwurm, A., David, P., Foschini, L., et al. 2003, A\&A, 411, L223 Labanti, C., Di Cocco, G., Ferro, G., et al. 2003, A\&A, 411, L149 Malaguti, G., Di Cocco, G., \& Stephen, J. B. 2001, Proc. SPIE, 3765 , 42

Malaguti, G., Bazzano, A., \& Bird, A. J. 2003, A\&A, 411, L173

Natalucci, L., Bird, A. J., Bazzano, A., et al. 2003, A\&A, 411, L209

Segreto, A., Labanti, C., Bazzano, A., et al. 2003, A\&A, 411, L215 